\title{
Effect of a feeder layer composed of mouse embryonic and human foreskin fibroblasts on the proliferation of human embryonic stem cells
}

\author{
HUA YANG ${ }^{1,2 *}$, YING QIU $^{1 *}$, XIANGHUI ZENG ${ }^{1}$, YAN DING $^{3}$, \\ JIANYE ZENG ${ }^{1}$, KEHUAN LU ${ }^{2}$ and DONGSHENG LI ${ }^{3}$ \\ ${ }^{1}$ Reproductive Medical Center of Nanning Second People's Hospital, Third Affiliated Hospital of Guangxi Medical University, \\ Nanning, Guangxi 530031; ${ }^{2}$ State Key Laboratory for Conservation and Utilization of Subtropical Agro-Bioresources, \\ Animal Reproduction Institute, Guangxi University, Nanning, Guangxi 530004; ${ }^{3}$ Life Science Research Institute, \\ Taihe Hospital, Shiyan, Hubei 442000, P.R. China
}

Received December 5, 2014; Accepted October 29, 2015

DOI: $10.3892 /$ etm.2016.3204

\begin{abstract}
The aim of the present study was to investigate the effects of feeder layers composed of various ratios of mouse embryonic fibroblasts (MEFs) and human foreskin fibroblasts (hFFs) on the growth of human embryonic stem cells (hESCs). In addition, the secretion levels of basic fibroblast growth factor (bFGF) by the feeder layers was detected. MEFs and hFFs were treated with mitomycin $\mathrm{C}$ and seeded onto gelatin-coated plates at a density of $1 \times 10^{8}$ cells/l. The hFFs and MEFs were combined and plated at the following ratios: 0:1, 1:2, 1:1, 2:1 and 1:0. The secretion of bFGF by the various $\mathrm{hFF} / \mathrm{MEF}$ ratio feeder layers was detected using an enzyme-linked immunosorbent assay. Subsequently, hESCs were cultured on top of the various feeder layers. The differences in the cellular morphology of the hESCs were observed using microscopy, and the expression levels alkaline phosphatase (AKP) and octamer-binding transcription factor 4 (OCT-4) were detected using immunohistochemical analysis as indicators of differentiation status. The results showed that the hFFs secreted substantial quantities of bFGF, while no bFGF was secreted by the MEFs. The clones
\end{abstract}

Correspondence to: Professor Jianye Zeng, Reproductive Medical Center of Nanning Second People's Hospital, Third Affiliated Hospital of Guangxi Medical University, 13 Dancun Road, Nanning, Guangxi 530031, P.R. China

E-mail: jianyezeng@126.com

Professor Kehuan Lu, State Key Laboratory for Conservation and Utilization of Subtropical Agro-Bioresources, Animal Reproduction Institute, Guangxi University, 100 Daxuedong Road, Nanning, Guangxi 530004, P.R. China

E-mail: kehuanlu@126.com

*Contributed equally

Key words: human embryonic stem cell, fibroblasts, feeder layer, basic fibroblast growth factor secretion of hESC growing on the feeder layer containing MEF or hFF alone were flat. By contrast, hESC clones grown on a mixed feeder layer containing hFFs + MEFs at a ratio of 1:1 exhibited an accumulated growth with a clear edge, as compared with the other ratios. In addition, hESCs growing on the feeder layer were positive for the expression of AKP and OCT-4. In summary, feeder layer hFFs secreted bFGF, while MEFs did not, indicating that bFGF is not the only factor that supports the growth and differentiation of hESCs. The optimal growth of hESCs was achieved using a mixed feeder layer composed of hFFs + MEFs at a ratio of 1:1.

\section{Introduction}

Human embryonic stem cells (hESCs) are a class of pluripotent cells isolated from the inner cell mass of human blastocysts. hESCs are hypothesized to be able to provide an unlimited cell source for the treatment of numerous refractory diseases in humans, including diabetes, leukemia, Parkinson's disease and juvenile rheumatoid arthritis (1). A number of preliminary and clinical studies have indicated that the repair of damaged tissues may be achieved via the transplantation of embryonic stem cells or their derived cells (2-4).

A key issue for the effective passage of hESCs is the inhibition of spontaneous differentiation and the maintenance of cell pluripotency. In previous studies, mouse embryonic fibroblasts (MEFs) and human foreskin fibroblasts (hFFs) have been used as feeder cells in a layer to support the growth of hESCs (5-8). Different cells in the feeding layers secrete various growth factors to support the pluripotency and non-differentiation of hESCs. The ability of a culture medium to support the growth of hESCs is evaluated by detecting the expression of hESC markers. However, at present, it is not known if the factors secreted by the feed layer have a role in maintaining the non-differentiation status of hESCs.

Cytokines that maintain the pluripotency, self-renewal and non-differentiation status of hESCs can be directly added to the culture medium, secreted by the feeding layers or activated by the feed layer cells. It has previously been demonstrated that 
it is challenging for a culture system without a feeding layer to maintain non-differentiation growth without the addition of exogenous cytokines. Cytokines secreted by the feeding layer are complicated and it is unknown which factors secreted by the feeding layer are able to promote the proliferation and inhibit the differentiation of hESCs. However, it is widely accepted that the addition of basic fibroblast growth factor (bFGF) is beneficial for the growth of hESCs; as such, bFGF has been widely applied in the hESC culturing system, in the presence and absence of a feeding layer $(9,10)$. Saxena et al (11) reported that bFGF was able to support the self-renewal of hESCs. In addition, self-renewal was achieved by activation of the phosphoinositide 3-kinase (PI3K)/Akt/protein kinase B (PKB) pathway and upregulation of integrin $\alpha 6 / \beta 1$.

In the present study the efficiencies of feeder layers composed of various ratios of MEFs and hFFs were compared with those of feeder layers composed of MEFs or hFFs alone. The aim was to develop a novel approach to solve the problems present in the regular culturing of hESCs.

\section{Materials and methods}

Materials. The present study was performed at the Reproductive Medical Center of Nanning Second People's Hospital (Nanning, China). Foreskin tissue samples were obtained from a 7-year-old boy who had previously undergone circumcision at the Nanning Second People's Hospital. Written informed consent was obtained froom the parents of the participant. The present study was approved by the ethics committee of Nanning Second People's Hospital. The hESC line NS-1 was isolated from human blastocysts, according to the method described in a previous study (12). Clean-level mice $(n=3)$ at 12.5-14.5 days of pregnancy were purchased from Beijing Vital River Laboratory Animal Technology Co., Ltd. (Beijing, China). Animal experiments were conducted in accordance with animal ethics standards of Nanning Second People's Hospital. Following anesthetization by abdominal injection with $40 \mathrm{mg} / \mathrm{kg}$ barbital (Guangdong Jiabo Pharmaceutical Co., Ltd., Guangzhou, China), the head, limbs and internal organs of the pregnant mice were removed. Cell suspension was obtained by conventional repeated trypsin (Sigma-Aldrich) digestion. Original cells were frozen once the cells were confluent. A feeder layer composed of MEFs was prepared by mitomycin $\mathrm{C}$ treatment, as described previously (9), for 2-3 $\mathrm{h}$ and inoculation onto a gelatin-coated dish (Sigma-Aldrich, St. Louis, MO, USA) at the density of $1 \times 10^{8}$ cells/1. Foreskin tissue was sterilized and cell suspension was obtained using trypsin digestion. Original cells were frozen once the cells were confluent. The feeder layers containing hFFs and mixed layers containing hFFs + MEFs were prepared in a similar manner.

Cellular characterization. Cellular morphology was observed using immunofluorescence staining, as described previously (10). Briefly, cells at generation 3-5 were obtained by digestion with $0.2 \%$ dispase (Sigma-Aldrich) and placed onto culturing dishes under coverslips. When $70-80 \%$ confluence was achieved, the cells were washed 2-3 times with Dulbecco's phosphate-buffered saline (DPBS; Sigma-Aldrich). The cells on the coverslips were then fixed with cold methanol $(75 \%)$ for $25 \mathrm{~min}$. After rinsing twice with DPBS, the cells were blocked with DPBS containing 1\% fetal bovine serum (FBS) for $40 \mathrm{~min}$. After rinsing twice with DPBS, the coverslips were incubated with primary antibodies, including rabbit anti-human keratin polyclonal antibody (1:100; E3260-1; Spring Bioscience Corporation, Pleasanton, CA, USA) and rabbit anti-humna vimentin polyclonal antibody (1:100; E4621-2; Spring Bioscience Corporation). Following incubation overnight at $37^{\circ} \mathrm{C}$ in $5 \% \mathrm{CO}_{2}$, the coverslips were rinsed 4 times with DPBS. Subsequently, the coverslips were incubated with fluorescein isothiocyanate (FITC)-conjugated goat anti-mouse IgG (1:100; E8921-0; Spring Bioscience Corporation) in the dark for 20-25 min. Following a further four rinses, propidium iodide was added and the coverslips were incubated in the dark for $15 \mathrm{~min}$. Following a further three rinses, images were obtained immediately using the DMIRE2 Fluorescent Microscope (Leica Microsystems $\mathrm{GmbH}$, Wetzlar, Germany). Fibroblasts exhibiting positive expression of vimentin were stained green. Negative staining for keratin indicated non-epithelial cells.

Detection ofbFGF secreted byfeeder layers. An enzyme-linked immunosorbent assay (ELISA; Fuzhou Maixin Biotechnology Development Co., Ltd., Fuzhou, China) was used to detect the concentration of bFGF secreted by the feeder layer cells. Known concentrations of bFGF in Dulbecco's modified Eagle's medium (DMEM) and DMEM/F12 medium (Gibco; Thermo Fisher Scientific, Inc., Waltham, MA, USA) were used to generate a standard curve. DMEM/F12 is designed for hESC culturing, and was supplemented with $20 \%$ KnockOut Serum Replacement formulation (SR; Thermo Fisher Scientific, Inc.), which resulted in an osmotic pressure similar to that of normal embryonic tissues. Following seeding of the hFFs or MEFs for $24 \mathrm{~h}$, the supernatant of the cells was isolated by centrifugation $\left(4^{\circ} \mathrm{C}, 256 \mathrm{x} \mathrm{g}\right)$, and the concentration of bFGF in the supernatant was detected using the ELISA. Following treatment with mitomycin $\mathrm{C}$, the concentration of bFGF was determined in the supernatant of the feeder layer cells with or without inoculation of hESC $(6,24$ and $72 \mathrm{~h})$. In addition, $4 \mathrm{ng} / \mathrm{ml}$ exogenous bFGF (Gibco; Thermo Fisher Scientific, Inc.) was added to the MEF feeder layer, in order to sustain hESC self-renewal (13).

Preparation of mixed feeder layers. The number of MEFs and hFFs were counted following mitomycin $\mathrm{C}$ treatment using the Countess II FL Automated Cell Counter (Thermo Fisher Scientific, Inc.). Subsequently, hFFs were mixed with MEFs at ratios of $0: 1,1: 2,1: 1,2: 1$ and 1:0 and seeded on gelatin-coated dishes at a density of $1 \times 10^{8}$ cells $/ 1$.

Passage of hESCs and morphological observations. The hESC clones were mechanically cut into small cell clumps (20-50 cells) using an attenuated Pasteur pipette (Sanofi Pasteur MSD Ltd., Maidenhead, UK). The cell clumps were then planted on the top of three feeder layers (MEFs, hFFs and hFFs + MEFs). The medium was changed every day and passage was made every 5-6 days. The clone morphology was observed and recorded using the CX41 phase contrast microscope (Olympus Corporation, Tokyo, Japan).

Detection of non-differentiated hESCs grown on the mixed feeder layers. The differentiation status was determined by 
A

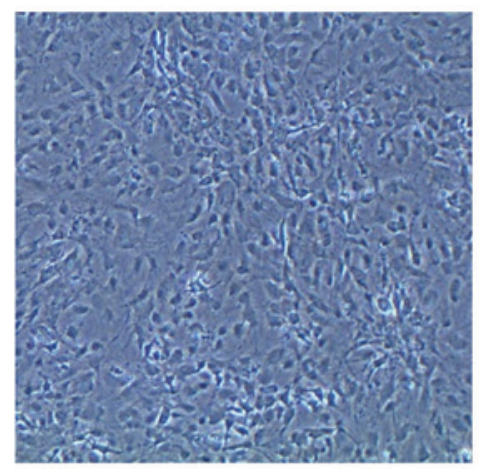

C

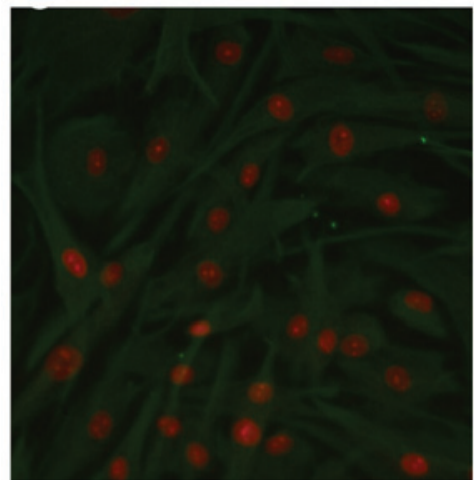

B

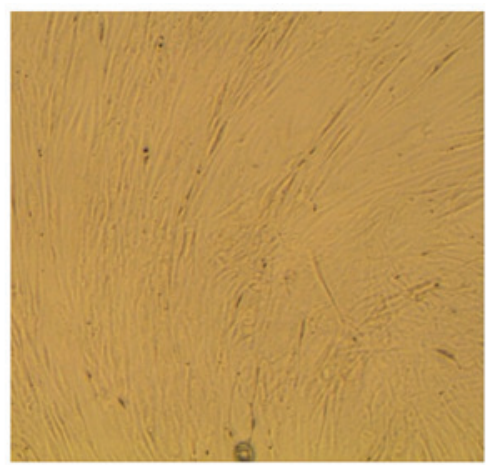

D

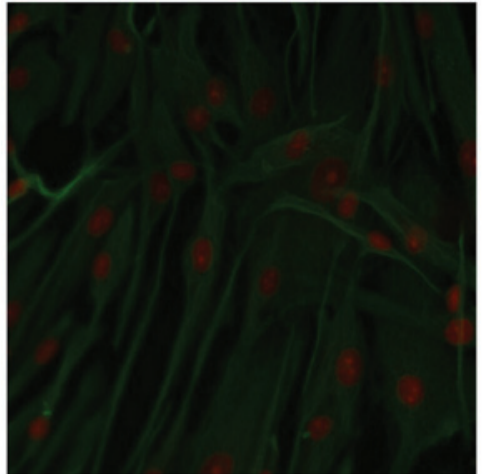

Figure 1. Characterization of mouse embryonic fibroblasts (MEFs) and human foreskin fibroblasts (hFFs) under immunofluorescence microscopy. Morphology of (A) MEFs and (B) hFFs under light microscopy. Fluorescence analysis of (C) MEFs and (D) hFFs stained with anti-vimentin antibody (green) and nucleus (red) (magnification, x100).

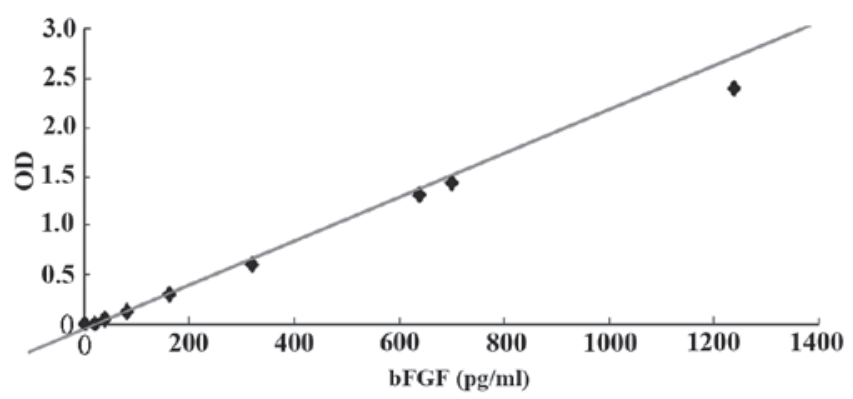

Figure 2. Concentration of bFGF in the medium of hFFs and/or MEFs. bFGF, basic fibroblast growth factor; hFF, human foreskin fibroblasts; MEFs, mouse embryonic fibroblasts.

detecting the expression of alkaline phosphatase (AKP) and octamer-binding transcription factor 4 (OCT-4). AKP was detected using a commercial kit (Fuzhou Maixin Biotechnology Development Co., Ltd.). Briefly, hESCs grown on the feeder layer for 5 days were fixed with $90 \%$ ethanol. After washing, AKP labeling reagents (Fuzhou Maixin Biotechnology Development Co., Ltd.) were added and the cells were incubated for $15 \mathrm{~min}$ in the dark. When the color was fully developed, the cells were visualized under the DVM6 Optical Microscope (Leica Microsystems GmbH).

For immunohistochemical detection of OCT-4, hESCs were washed with PBS and fixed with $40 \mathrm{~g} / 1$ paraformaldehyde for $15 \mathrm{~min}$. Subsequently, the cells were incubated for $40 \mathrm{~min}$ at room temperature with rabbit anti-human OCT-4 polyclonal antibody (1:50; ab19857; Abcam, Cambridge, MA, USA). Following washing with PBS, the cells were incubated for $40 \mathrm{~min}$ at room temperature with FITC-conjugated goat

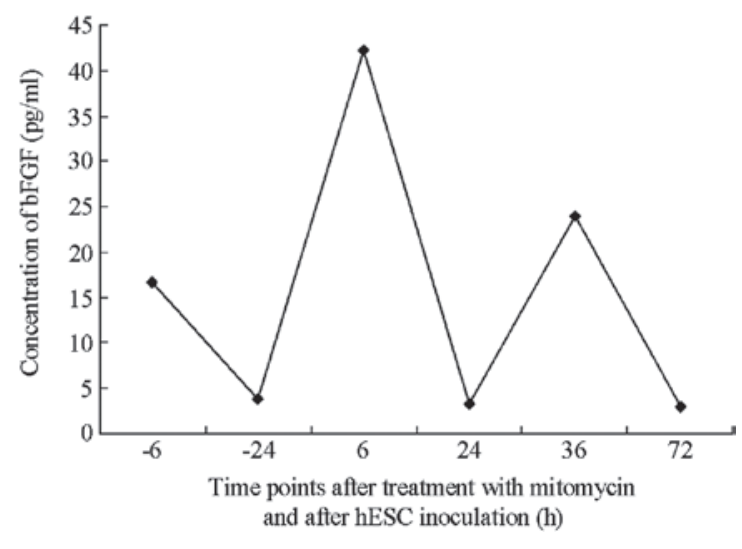

Figure 3. Dynamic concentration of bFGF in the human foreskin fibroblast feeder layer. Y-axis represents the concentration of bFGF and X-axis represents the time points following the treatment with mitomycin C. bFGF, basic fibroblast growth factor; hESC, human embryonic stem cell.

anti-rabbit IgG (1:200; ab21321, Abcam). OCT-4 expression was detected using the DMIRE2 Fluorescent Microscope. hESC clones positive for OCT-4 exhibited a green color.

The mRNA expression levels of OCT-4 were determined using a reverse transcription-polymerase chain reaction (RT-PCR), according to a previous study (14). Briefly, RNA was isolated from hESCs using TRIzol ${ }^{\circledR}$ reagent (Invitrogen; Thermo Fisher Scientific, Inc.). Reverse transcription was performed using $1 \mu \mathrm{g}$ RNA and the PrimeScript ${ }^{\mathrm{TM}}$ RT Reagent kit (Takara Biotechnology Co., Ltd., Dalian, China). PCR was conducted in a C1000 Touch $^{\text {TM }}$ thermal cycler (Bio-Rad Laboratories, Inc., Hercules, CA, USA), using the PCR Master Mix (Promega Corporation, Madison, WI, USA) with $1 \mu 1$ 


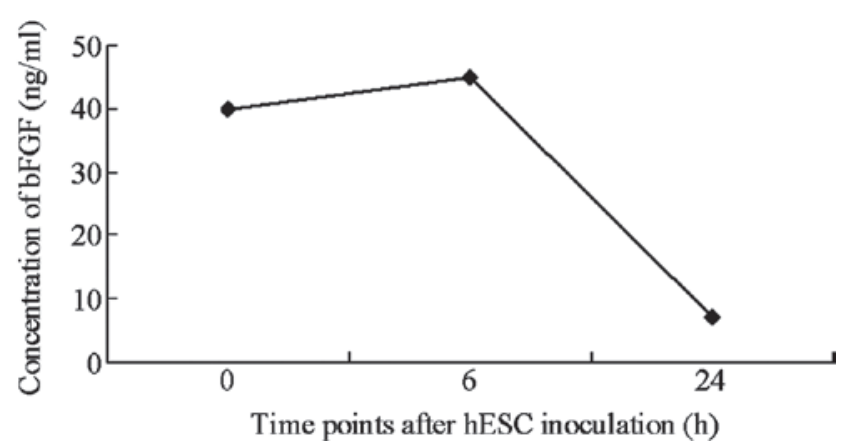

Figure 4. Dynamic concentration of bFGF in the mouse embryonic fibroblasts inoculated with hESCs. Y-axis represents the concentration of bFGF and the $\mathrm{X}$-axis represents the time points following hESC inoculation. bFGF, basic fibroblast growth factor; hESC, human embryonic stem cell.

cDNA, $1 \mu \mathrm{l}$ each of sense and anti-sense primers, and $5 \mu 1 \mathrm{H}_{2} \mathrm{O}$. The PCR cycling conditions were as follows: $95^{\circ} \mathrm{C}$ for $30 \mathrm{sec}$, followed by 32 cycles of $95^{\circ} \mathrm{C}$ for $30 \mathrm{sec}, 60^{\circ} \mathrm{C}$ for $30 \mathrm{sec}$ and $72^{\circ} \mathrm{C}$ for $30 \mathrm{sec}$. The primer sequences were as follows: OCT- 4 sense, 5'-GACAACAATGAGAACCTTCAGGAGAA-3' and anti-sense, 5'-TTCTGGCGCCGGTTACAGAACCA-3'; and GAPDH sense, 5'-GTCAGTGGTGGACCTGACCT-3' and anti-sense, 5'-CACCACCCTGTTGCTGTAGCA-3' (Sangon Biotech, Co., Ltd., Shanghai, China). The fold-changes in gene expression were normalized to GAPDH. PCR products were separated by $2 \%$ agarose gel electrophoresis (Sigma-Aldrich) and visualized using the U-2900 UV-Vis Double Beam Spectrophotometer (Hitachi, Ltd., Tokyo, Japan). The gel images were analyzed using ImageJ 2x software (National Institutes of Health, Bethesda, MA, USA).

In vitro differentiation experiment. hESC clones $\left(1 \times 10^{6}\right)$ were transferred onto culturing dishes without feeder layers. The clones were cultured in embryonic stem cell medium (Fuzhou Maixin Biotechnology Development Co., Ltd.) without the basic components of fibroblast growth factors for 7-10 days. Morphology was observed under a microscope.

Statistical analysis. All statistical analyses were conducted using SPSS software, version 17.0 (SPSS, Inc., Chicago, IL, USA). Data are presented as the mean \pm standard deviation. The differences between two groups were compared using the Student's unpaired t-test. $\mathrm{P}<0.05$ was considered to indicate a statistically significant difference.

\section{Results}

Characterization of MEFs and hFFs by anti-vimentin fluorescence analysis. Anti-vimentin staining showed that MEFs and hFFs obtained via standard procedure were positive for vimentin. Since vimentin has been shown to be a marker of mesenchymal cells (15), these results indicated the presence of non-epithelial cells (Fig. 1).

Secretion of bFGF by hFFs. An ELISA indicated that the content of bFGF in the hFF feeder layer medium was not altered by the presence of mitomycin $\mathrm{C}$ or by the replacement of FBS with SR. The absence of bFGF in the FBS and SR
$\mathbf{A}$

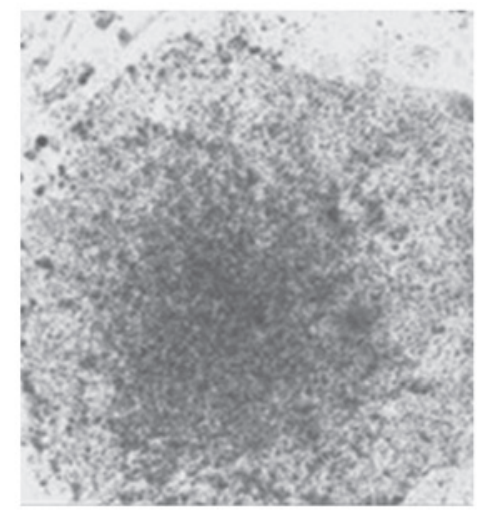

B

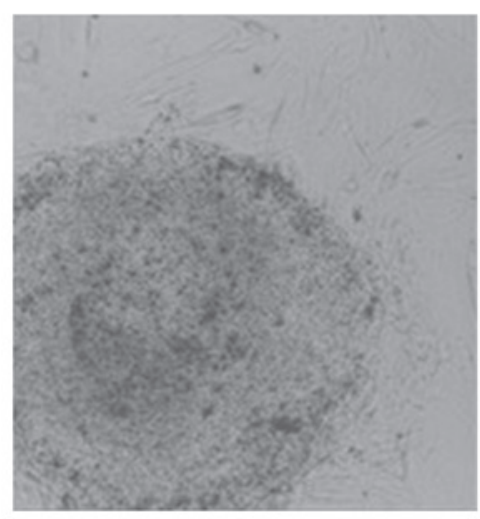

C

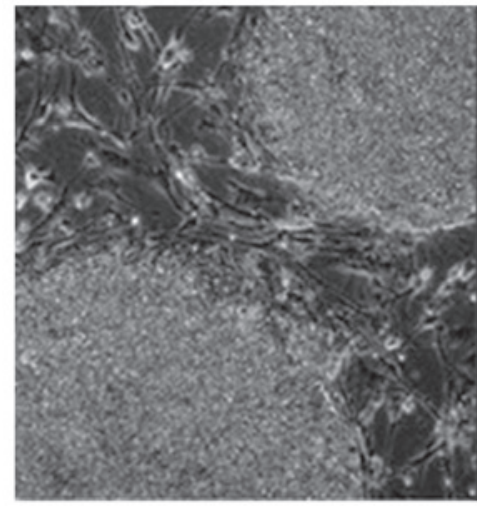

Figure 5. Morphology of human embryonic stem cells grown on the feeder layer of (A) mouse embryonic fibroblasts (MEFs), (B) human foreskin fibroblasts (hFFs) and (C) MEFs + hFFs.

indicated that the bFGF detected in the medium had been secreted by the feeder layer. Furthermore, mitomycin $\mathrm{C}$ treatment did not inhibit the autonomous secretion of bFGF (Figs. 2 and 3). In addition, the maximal autonomous secretion of bFGF was achieved in the first passage of feeder layer cell inoculation. Following the adherent growth of hESCs, the secretion of bFGF decreased significantly, suggesting that bFGF was crucially involved in facilitating the adherent cell growth. Furthermore, bFGF was mildly increased at the first passage of hESC inoculation into the mixed feeder layer. The ratio of hFFs to MEFs was 0:1, 1:2, 1:1, 2:1 and 1:0.

MEFs do not secrete detectable levels of bFGF. bFGF content was rapidly reduced following the adherent growth of hESCs. After $24 \mathrm{~h}$ of growth, bFGF content in the medium was reduced to $7 \mathrm{pg} / \mathrm{ml}$. Gradual reductions in $\mathrm{bFGF}$ and 
A

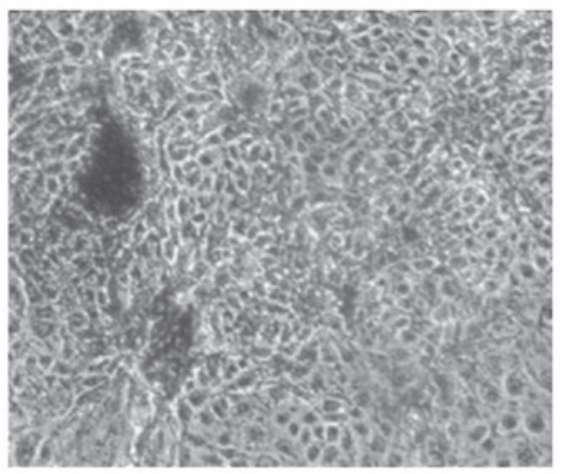

C

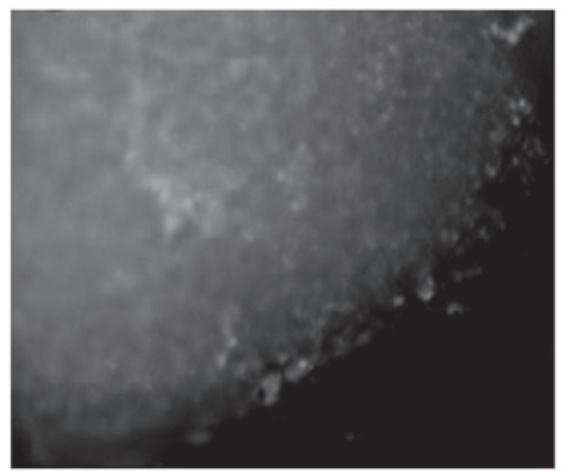

B

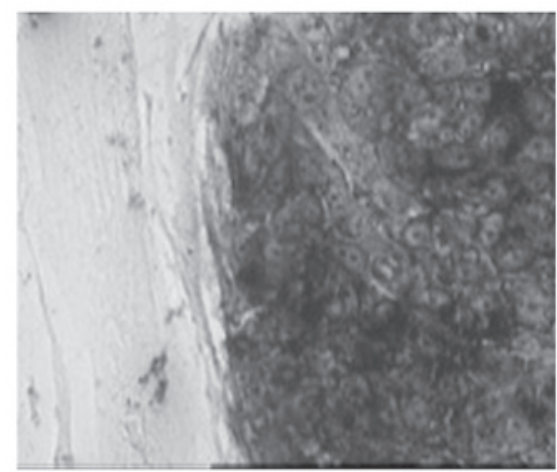

D

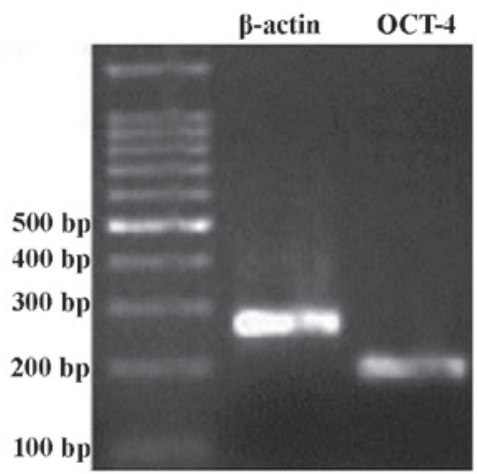

Figure 6.Characterization of human embryonic stem cells by (A and B) alkaline phosphatase staining,(C) OCT-4 staining and (D) reverse transcription-polymerase chain reaction analysis of OCT-4 (magnification, x100). OCT-4, octamer binding transcription factor- 4 .

A

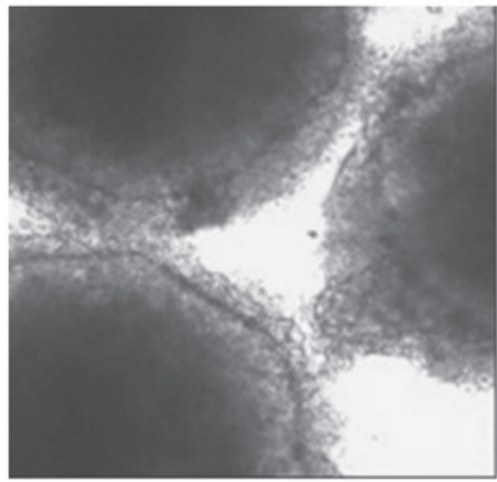

B

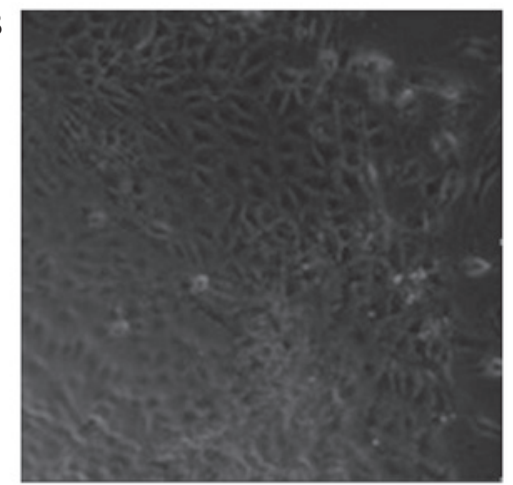

Figure 7. Morphology of (A) embryonic bodies and (B) differentiated adherent cells.

significant increases in hESC proliferation indicated that bFGF was utilized by the hESCs. bFGF is the most common additive in hESC culture media and is able to markedly enhance the proliferation of hESCs. Fibroblasts express multiple types of bFGF, while hESCs express multiple types of $\mathrm{bFGF}$ receptor. The present results suggest that bFGF is able to enhance cell adherence and clone formation rate in the initial stages of hESC clone development and support the proliferation of hESCs (Fig. 4). These results are consistent with the commonly accepted hypothesis that bFGF is beneficial for the growth of hESCs.

Comparison of hESC growth between different feeder layers. The hESCs grown on the MEF (0:1) feeder layer had a clear clonal edge with distinct boundary with the surrounding cells. However, the clones were flat without obvious upheaving (Fig. 5A). These clones appeared 'thin' (Fig. 5A). The hESCs grown on the hFF (1:0) feeder layer exhibited a clear boundary with the surrounding cells. However, the clones were not plump and no cellular accumulation was observed. In addition, the center of the clonal mass was prone to differentiation. In addition, the clones appeared 'thin' (Fig. 5B). The hESCs grown on the mixed (1:1) feeder layer containing hFFs + MEFs had a clear boundary, significant upheaving and exhibited accumulative growth. Overall, the clones appeared 'thick'. The morphology of hESCs grown on the mixed (1:1) feeder layer was improved compared with those grown on the MEF (0:1) or hFF (1:0) feeder layers (Fig. 5C).

Growth of hESCs among the mixed feeder layers with various ratios of MEF and $h F F$. In the feeder layer containing 
hFFs + MEFs at a ratio of 1:0, hESCs exhibited a clear clonal boundary; however, the clones were flat without obvious upheaving. Similar morphology was observed in the feeder layer containing $\mathrm{hFF}+\mathrm{MEFs}$ at a ratio of 1:2. In the feeder layer containing hFFs + MEFs at a ratio of 1:1, the hESCs exhibited a clear clonal boundary and accumulative growth. In addition, the clones had clear upheaving and were plump and the cells had tight connections. Similar results were observed when the ratio of MEF and hFF was 1:2. When the ratio of MEF and $\mathrm{hFF}$ was $0: 1$, the edge of the $\mathrm{hESC}$ clones was evident. However, the clones were not plump, with no obvious upheaving, and the center was prone to differentiation.

Detection of non-differentiation status in the hESCs grown on the mixed feeder layers. The feeder layer cells and differentiated cells were negative for AKP staining (Fig. 6A). hESCs grown on the mixed feeder layers were stained dark purple by the AKP staining, indicating a marked positive levels of AKP expression, and that these cells were non-differentiated (Fig. 6B). Immunohistochemical analysis for the detection of OCT- 4 showed that non-differentiated hESCs exhibited a green color, indicating that OCT-4 was markedly expressed (Fig. 6C). The results of the RT-PCR analysis showed that OCT-4 mRNA expression was present in the non-differentiated hESCs (Fig. 6D).

Formation of embryonic bodies. Following the removal of the feeder layer, the remaining hESCs were able to form embryonic bodies in the suspension cultures (Fig. 7A). Following the achievement of adherence, the hESCs were able to differentiate into cells with multiple morphologies. Normal hESCs had large nuclei and a high nuclear-cytoplasmic ratio. When induced to differentiate in vitro, they were able to form the endoderm, desoderm and ectoderm layers (Fig. 7B).

\section{Discussion}

Different cell types in feeder layers are able to secrete various growth factors to maintain the pluripotency and non-differentiation of growth of hESCs. Currently, the ability of the culture medium to support the growth of hESCs is evaluated by detecting the expression of markers on the hESCs (16). However, it remained unclear whether the factors secreted by the feeder layer were involved in the maintenance of non-differentiation status of hESCs. Cytokines that maintain the pluripotency, self-renewal and non-differentiation status of hESCs may be directly added to the medium, or secreted by the feeder layers, or activated by the feed layer cells. It is difficult for the culture system alone, without a feeder layer, to maintain non-differentiation growth if exogenous cytokines are not added (17). The cytokine secretion profiles of feeder layers are complicated, and it is unknown what factors secreted by the feeder layer are involved in promoting the proliferation and inhibition of hESC differentiation. It is commonly accepted that the addition of bFGF is beneficial for the growth of hESCs, and bFGF has been widely applied in hESC culturing systems with or without a feeder layer (18). A previous study demonstrated that bFGF promotes the proliferation of hESCs by binding to the bFGF receptors expressed on
hESCs, resulting in the upregulation of HLA class I molecules, the downregulation of human leukocyte antigen-DR and the activation of genes associated with the cell proliferation (19). Saxena et al (11) showed that the ability of bFGF to support the self-renewal of hESCs was necessary. In addition, self-renewal was achieved by the activation of the PI3K/PKB pathway and upregulation of integrin $\alpha 6 / \beta 1$.

The results of the present study show that the MEF (0:1) feeder layer did not secrete detectable levels of bFGF. By contrast, the hFF (1:0) feeder layer secreted bFGF. After $6 \mathrm{~h}$ of inoculation, bFGF expression was detectable in the hFF tissues. The quantity of bFGF secreted by the hFFs after $24 \mathrm{~h}$ of inoculation was not significantly different compared with after 3 days of inoculation, suggesting that an hFF feeder layer may be utilized after 3 days of treatment with mitomycin C. These results are consistent with a previous report (20), which demonstrated that an hFF feeder layer is able to survive for an extended period following treatment with mitomycin C. Thus, hFFs may be superior to MEFs for use in feeder layers to support the growth of hESCs. In the present study, exogenous bFGF (optimal concentration, $4 \mathrm{ng} / \mathrm{ml}$ ) was added to the MEF feeder layer, while bFGF was not added to the hFF feeder layer. The results showed that hESCs exhibited marked adherent growth on MEF and hFF feeder layers. However, the non-differentiation rate of hESCs on the hFF feeder layer was reduced compared with that of hESCs grown on the MEF feeder layer.

Previous studies have evaluated the impact of bFGF on the non-differentiation growth of hESCs in an MEF culturing system $(21,22)$. Zhou et al (21) showed that bFGF induced MEF in a concentration-dependent manner, and the optimal concentration of bFGF to induce MEFs was $4 \mathrm{ng} / \mathrm{ml}$. In addition, bFGF functions in MEFs, but not hESCs. These results demonstrate that bFGF is necessary for MEFs to support the non-differentiation growth of hESCs. Xu et al (22) showed that hESCs are able to maintain stable proliferation, pluripotency and non-differentiation status if grown on an MEF feeder layer supplemented with 160 or $250 \mathrm{ng} / \mathrm{ml}$ bFGF. Therefore, the microenvironment of the different feeder layers varied. Currently, the optimal concentration of bFGF required to support the growth of hESCs is unknown. The function of bFGF may be affected by its source (secreted or exogenous), purity, concentration and the expression of bFGF receptor in hESCs.

It remains unclear whether the inhibition of mitosis by mitomycin C (23) may promote the secretion of new proteins or alter the secretion of growth factors by feeder layer cells. In the present study, mitomycin $\mathrm{C}$ treatment did not appear to inhibit the secretion of bFGF by hFFs. The peak bFGF content was detected during the initial stage of hESC inoculation in the $\mathrm{hFF}$ feeder layer. Following the adherent growth of hESCs, the bFGF content was significantly reduced. The concentration of bFGF in the MEF culturing system containing $4 \mathrm{ng} / \mathrm{ml}$ exogenous $\mathrm{bFGF}$ was reduced to $7 \mathrm{pg} / \mathrm{ml}$ after $24 \mathrm{~h}$ of hESC growth. Thus, it was hypothesized that bFGF is able to enhance the adherence and clone formation rate and support the proliferation of hESCs at the initial stages of growth. In addition, bFGF content was moderately increased during the initial period of $\mathrm{hESC}$ inoculation in the MEF and hFF feeder layers, which is consistent with a previous study (24), indicating that hESCs 
are able to regulate their own self-renewal and facilitate the secretion of bFGF.

In previous studies involving hESC lines and in vitro culture, fibroblasts obtained from fetal mice at 12.5-14.5 days pregnancy were used as feeder cells to support the growth of hESCs $(25,26)$. Furthermore, a number of cell types have been reported to be able to support hESC growth in vitro, including hFFs (5-8), human endometrial cells (27), human placental fibroblasts (28), human fetal skin cells (29) and hESC-derived fibroblasts (30-32). In the present study, MEFs and hFFs were shown to be able to support hESC growth in vitro; however, the common problem remained that the density of hESCs was low.

In conclusion, the present study combined MEFs and hFFs at various ratios $(1: 1,2: 1$ and $1: 2)$ to produce a mixed feeder layer in order to support the growth of hESCs in vitro. The results indicated that the mixed feeder layer was able to promote the growth of hESCs, with the hESCs remaining in an undifferentiated state. Furthermore, the results suggested that a hFFs + MEFs feeder layer at a ratio of 1:1 and 1:2 produced comparable results, and were significantly more supportive of cellular growth, as compared with non-mixed feeder layers. This may be due to the weak adherence of MEFs, as compared with hFFs. Since the MEFs after passage five were unable to support the proliferation of hESCs, it may be that the factors required for stem cell growth were predominantly derived from the hFFs. The mixed feeder layer established in the present study may not only support the growth of hESCs in vitro, but also replace the conventional single cell feeder layer.

\section{Acknowledgements}

The present study was supported by the Scientific and Technological Project of Guangxi Province (grant no. 0993003A-23) and the Major Science and Technology of Nanning (grant no. 200801024C).

\section{References}

1. Whyte M, Hubbard R, Meliconi R, Whidborne M, Eaton V, Bingle C, Timms J, Duff G, Facchini A, Pacilli A, et al: Increased risk of fibrosing alveolitis associated with interleukin-1 receptor antagonist and tumor necrosis factor-alpha gene polymorphisms. Am J Respir Crit Care Med 162: 755-758, 2000.

2. Bjorklund LM, Sánchez-Pernaute S, Chung S, Andersson T, Chen IY, McNaught KS, Brownell AL, Jenkins BG, Wahlestedt C, Kim KS and Isacson O: Embryonic stem cells develop into functional dopaminergic neurons after transplantation in a Parkinson rat model. Proc Natl Acad Sci USA 99 2244-2349, 2002.

3. Brüstle O, Jones KN, Learish RD, Karram K, Choudhary K, Wiestler OD, Duncan ID and McKay RD: Embryonic stem cell-derived glial precursors: A source of myelinating transplants. Science 285: 754-756, 1999

4. Liu S, Qu Y, Stewart TJ, Howard MJ, Chakrabortty S, Holekamp TF and McDonald JW: Embryonic stem cells differentiate into oligodendrocytes and myelinate in culture and after spinal cord transplantation. Proc Natl Acad Sci USA 97: 6126-6131, 2000.

5. Amit M, Margulets V, Segev H, Shariki K, Laevsky I, Coleman R and Itskovitz-Eldor J: Human feeder layers for human embryonic stem cells. Biol Reprod 68: 2150-2156, 2003.

6. Hovatta O, Mikkola M, Gertow K, Strömberg AM, Inzunza J, Hreinsson J, Rozell B, Blennow $\mathrm{E}$, Andäng $\mathrm{M}$ and Ahrlund-Richter L: A culture system using human foreskin fibroblasts as feeder cells allows production of human embryonic stem cells. Hum Reprod 18: 1404-1409, 2003.
7. Inzunza J, Gertow K, Strömberg MA, Matilainen E, Blennow E, Skottman H, Wolbank S, Ahrlund-Richter L and Hovatta O: Derivation of human embryonic stem cell lines in serum replacement medium using postnatal human fibroblasts as feeder cells. Stem Cells 23: 544-549, 2005.

8. Nieto A, Cabrera CM, Catalina P, Cobo F, Barnie A, Cortés JL, Barroso del Jesus A, Montes R and Concha A: Effect of mitomycin- $\mathrm{C}$ on human foreskin fibroblasts used as feeders in human embryonic stem cells: Immunocytochemistry MIBI score and DNA ploidy and apoptosis evaluated by flow cytometry. Cell Biol Int 31: 269-278, 2007.

9. Thomson JA, Itskovitz-Eldor J, Shapiro SS, Waknitz MA, Swiergiel JJ, Marshall VS and Jones JM: Embryonic stem cell lines derived from human blastocytes. Science 282: 1145-1147, 1998.

10. Troy TC, Rahbar R, Diker B and Turksen K: Immunolocalization in the epidermis. Methods Mol Biol 289: 113-120, 2005.

11. Saxena S, Hanwate M, Deb K, Sharma V and Totey S: FGF2 secreting human fibroblast feeder cells: A novel culture system for human embryonic stem cells. Mol Reprod Dev 75: 1523-1532, 2008.

12. Gavrilov S, Marolt D, Douglas NC, Prosser RW, Khalid I, Sauer MV, Landry DW, Vunjak-Novakovic G and Papaioannou VE: Derivation of two new human embryonic stem cell lines from nonviable human embryos. Stem Cells Int 2011: 765378, 2011

13. Vaajasaari H, Ilmarinen T, Juuti-Uusitalo K, Rajala K, Onnela N, Narkilahti S, Suuronen R, Hyttinen J, Uusitalo H and Skottman H: Toward the defined and xeno-free differentiation of functional human pluripotent stem cell-derived retinal pigment epithelial cells. Mol Vis 17: 558-575, 2011.

14. Ding Y, Yang H, Yu L, Xu CL, Zeng Y, Qiu Y and Li DS: Feeder-free and xeno-free culture of human pluripotent stem cells using UCBS matrix. Cell Biol Int 39: 1111-1119, 2015.

15. Coulombe PA and Wong I: Cytoplasmic intermediate filaments revealed as dynamic and multipurpose scaffolds. Nat Cell Biol 6:699-706, 2004.

16. Xu C, Inokuma MS, Denham J, Golds K, Kundu P, Gold JD and Carpenter MK: Feeder-free growth of undifferentiated human embryonic stem cells. Nat Biotechnol 19: 971-974, 2001.

17. Hirai H, Karian P and Kikyo N: Regulation of embryonic stem cell self-renewal and pluripotency by leukaemia inhibitory factor. Biochem J 438: 11-23, 2011.

18. Salli U, Fox TE, Carkaci-Salli N, Sharma A, Robertson GP, Kester M and Vrana KE: Propagation of undifferentiated human embryonic stem cells with nano-liposomal ceramide. Stem Cells Dev 18: 55-65, 2009.

19. Yabut $\mathrm{O}$ and Bernstein HS: The promise of human embryonic stem cells in aging-associated diseases. Aging (Albany NY) 3: 494-508, 2011.

20. Huang YC, Wang TW, Sun JS and Lin FH: Investigation of mitomycin-C-treated fibroblasts in 3-D collagen gel and conditioned medium for keratinocyte proliferation. Artif Organs 30: 150-159, 2006.

21. Zhou YP, Rochat A, Hatzfeld A, Peiffer I, Barbet R, Hatzfeld J and Li ML: bFGF-stimulated MEF-conditioned medium is capable of maintaining human embryonic stem cells. Fen $\mathrm{Zi} \mathrm{Xi}$ Bao Sheng Wu Xue Bao 42: 193-199, 2009 (In Chinese).

22. Xu HF and Suming Z: Feeder-free growth of human embryonic stem cells supported by basic fibroblast growth factor. Zhong Guo Zu Zhi Gong Cheng Yan Jiu Yu Lin Chuang Kang Fu 14: 1111-1114, 2010 (In Chinese).

23. Chiba S, Lee YM, Zhou W and Freed CR: Noggin enhances dopamine neuron production from human embryonic stem cells and improves behavioral outcome after transplantation into Parkinsonian rats. Stem Cells 26: 2810-2120, 2008.

24. Eiselleova L, Peterkova I, Neradil J, Slaninova I, Hampl A and Dvorak P: Comparative study of mouse and human feeder cells for human embryonic stem cells. Int J Dev Biol 52: 353-363, 2008.

25. Li SS, Liu YH, Tseng CN, Chung TL, Lee TY and Singh S: Characterization and gene expression profiling of five new human embryonic stem cell lines derived in Taiwan. Stem Cells Dev 15: 532-555, 2006.

26. Mandal A, Tipnis S, Pal R, Ravindran G, Bose B, Patki A, Rao MS and Khanna A: Characterization and in vitro differentiation potential of a new human embryonic stem cell line, ReliCellhES1. Differentiation 74: 81-90, 2006.

27. Lee JB, Lee JE, Park JH, Kim SJ, Kim MK, Roh SI and Yoon HS: Establishment and maintenance of human embryonic stem cell lines on human feeder cells derived from uterine endometrium under serum-free condition. Biol Reprod 72: 42-49, 2005. 
28. Genbacev O, Krtolica A, Zdravkovic T, Brunette E, Powell S, Nath A, Caceres E, McMaster M, McDonagh S, Li Y, et al: Serum-free derivation of human embryonic stem cell lines on human placental fibroblast feeders. Fertil Steril 83: 1517-1529, 2005

29. Richards M, Tan S, Fong CY, Biswas A, Chan WK and Bongso A: Comparative evaluation of various human feeders for prolonged undifferentiated growth of human embryonic stem cells. Stem Cells 21: 546-556, 2003.

30. Xu C, Jiang J, Sottile V, McWhir J,Lebkowski J and Carpenter MK: Immortalized fibroblast-like cells derived from human embryonic stem cells support undifferentiated cell growth. Stem Cells 22: 972-980, 2004
31. Wang Q, Fang ZF, Jin F, Lu Y, Gai H and Sheng HZ: Derivation and growing human embryonic stem cells on feeders derived from themselves. Stem Cells 23: 1221-1227, 2005.

32. Stojkovic P, Lako M, Stewart R, Przyborski S, Armstrong L, Evans J, Murdoch A, Strachan T and Stojkovic M: An autogeneic feeder cell system that efficiently supports growth of undifferentiated human embryonic stem cells. Stem Cells 23: 306-314, 2005. 